\title{
Electrochemical synthesis and characterization of single-walled carbon nanotubes/polypyrrole films on transparent substrates.
}

\author{
Javier Hernández-Ferrer*, Alejandro Ansón-Casaos, Ma Teresa Martínez. \\ Instituto de Carboquímica (CSIC), C/ Miguel Luesma Castán 4, E-50018 Zaragoza,
} Spain.

* Corresponding author. Tel.: +34 976 733977; fax: +34 976733318

E-mail address: jhernandez@icb.csic.es (J. Hernández-Ferrer)

\begin{abstract}
:
Polypyrrole films were electrosynthesized potentiodinamically on transparent fluorinedoped tin dioxide (FTO) substrates. Polymerization was carried out in the presence and in the absence of sodium dodecylbenzenesulfonate (SDBS) and single-walled carbon nanotubes (SWCNTs). The effect of the presence of SDBS and the addition of both asgrown and air-oxidized SWCNTs to the polymerization solution was studied. Electrochemical techniques, as well as ex-situ techniques were used for film characterization. The results show that the slowly growing Ppy coating in course of the electropolymerization entrapped the SWCNTs, which are primarily attached to the electrode by Brownian motion and/or electrophoresis, in the polymer matrix. The presence of SDBS and SWCNTs lowers electrode impedance modulus, especially at
\end{abstract}


low frequencies, and increases film capacitance. The presence of SWCNTs increases thickness, roughness and mechanical stiffness of the films

Keywords: Polypyrrole electropolymerization, single-walled carbon nanotubes, electrochemical impedance spectroscopy, composite materials, nanotube purification.

\section{Introduction}

Polypyrrole (Ppy) and its composites have been electrochemically synthesized and studied under a very wide variety of conditions [1] and have shown to be promising materials for a large number of applications such as supercapacitors [2], cell growth [3], solar cells [4-6], corrosion protection [7, 8] or sensing [9], among others. Electrodeposition of Ppy on transparent substrates has been proposed as a good way of achieving proper substrates for cell growth since Ppy films exhibit good adhesion properties towards the cells and good biocompatibility, and their properties are tunable by varying the electrochemical conditions of the synthesis $[1,10]$.

Electrosynthesis of Ppy in water produces films with poorer electrical and mechanical properties than other solvents, such as nitromethane or acetonitrile[10], but their toxicity makes them unsuitable for biomedical applications. The electrosynthesis of Ppy can be favored by adjusting electrolyte ionic strength and $\mathrm{pH}$; in this case, a high electrolyte concentration and a neutral or slightly acid $\mathrm{pH}$ value increases the quality of the Ppy film [10]. Another way of improving the quality of electrodeposited polymer films is 
through the addition of doping agents $[11,12]$ that improve the electrical and/or mechanical properties of the material. Also, the electrochemical method employed in the electropolymerization process strongly affects the properties of the obtained film. In this paper, Cyclic Voltammetry was chosen as the electrochemical synthesis method instead of galvanostatic or potentiostatic methods mainly for two reasons: it is known to yield smoother films [13] and it is possible to monitor the redox properties of the deposits during the experiment, while galvanostatic and potentiostatic polymerizations allow the evaluation of the electrochemical properties only in the end of the experiment.

Despite their good electrical properties, Ppy films show weak mechanical properties that can be improved by the addition of carbon nanotubes, as has been observed previously for several polymeric matrixes [14-16], thus obtaining a more resistant and durable support. Nanotubes also improve the electrical conductivity of polymer films, thus lowering the electrical resistance and impedance modulus of these materials [17]. The electrochemical stability of polypyrrole is also improved by the addition of carbon nanotubes [18]. A low impedance modulus of the substrates is crucial in the study of the effects of electrical stimulation on cell growth $[19,20]$ and implantable electrodes $[21-$ 23].

Carbon nanotubes have been widely employed in electrochemical formation of composites with conducting polymers. However, the vast majority of electrochemical studies on these composites have been carried out using heavily oxidized CNTs [23, 24], which is mainly due to the presence of negatively charged oxygenated functional groups on the surface of the nanotubes enhancing their hydrophilicity and their 
interaction with the substrate electrode surface, given that electropolymerization is an oxidation reaction $[23,24]$. The absence of these surface groups in the as-grown nanotubes makes them poor candidates owing to both their dispersion in water and their electrophoretic migration towards the electrode.

Recent works [25-30] have shown that both as-grown and air-oxidized nanotubes can be purified using a method consisting of two steps: the first is dispersion in a surfactant or polymer solution and the second is the centrifugation and subsequent decantation of the sample to obtain a nanotube suspension of higher quality. One of the most widely used surfactants is sodium dodecylbenzenesulfonate (SDBS), which stabilizes water suspensions of carbon nanotubes $[25,28]$ and also acts as a dopant anion; that is to say, a molecule that, added to the electropolymerization solution, improves the electrochemical properties, of the obtained polymer [31]. Moreover, the negative charge of the sulfonate group will induce an electrophoretic motion towards the anode [32]. Additionally, adhesion between pyrrole and carbon nanotubes can be improved owing to the presence of the dopant anion on the nanotube surface.

The characterization of the electrical properties of the conducting polymer thin layer is usually performed using in-situ methods, such as Cyclic Voltammetry (CV) or Electrochemical Impedance Spectroscopy (EIS), which allow the evaluation of electrochemical properties of the system. $\mathrm{CV}$ is essential for the identification and characterization of redox processes occurring on the electrode surfaces. EIS is a very useful tool for obtaining electrochemical information on intrinsic capacitance, kinetic reactions or diffusion coefficients, as well as on the solution-film interface. However, 
the evaluation of intrinsic electronic conductivity of the polymer by EIS is very difficult to discern from other charge transfer phenomena within the polymer. In-situ direct measurements require a microelectrode array and the use of a bi-potentiostat. Furthermore, they are imprecise owing to the electronic characteristics of the setup, meaning that direct ex-situ measurements must also be made.

To be a proper substrate for cell growth, a film should be biocompatible, have low electrical impedance modulus between 100 and $1000 \mathrm{~Hz}$ and high mechanical stability. Furthermore, its thickness and transparency must be controlled, as well as be as uniform as possible. SDBS shows low toxicity and it's a well-known dopant ion. Moreover, it's able to disperse any type of SWCNT in an aqueous solution. So, the electrosynthesis of Ppy-SWCNT films in the presence of SDBS is a promising way in this direction.

This paper investigates the effect of the concentration and surface structure (as-grown or air-oxidized) of SDBS-purified SWCNTs on the properties of Ppy films, This type of study has not been reported so far, and it is a key point in the deep knowledge and fine design of the Ppy composites. Films are synthesized by electrodeposition on FTO in the presence of SDBS as a dopant anion. The change in the electrochemical, morphological and mechanical properties produced by the surfactant, the nanotubes, or the combination of both, is exhaustively studied using different techniques, such as CV, EIS, Scanning Electron Microscopy (SEM), Confocal Microscopy and Atomic Force Microscopy (AFM).

\section{Experimental}


Sodium monohydrogenophosphate heptahydrate, sodium dihydrogenophosphate monohydrate (puriss. p.a.), sodium dodecylbenzenesulfonate (technical grade), yttrium (99.9\%) and graphite powder ( $\geq 99.99 \%)$ were purchased from Aldrich (website http://www.sigmaaldrich.com). Nickel powder (99.9\%) was purchased from AlfaAesar (website http://www.alfa.com). Graphite bars were obtained from CYMIT Química, Barcelona, Spain. Pyrrole (SAFC, $\geq 98 \%$ FCC) was used without any further purification. Ultrapure water employed in the preparation of the solutions was obtained from a Milli-Q system from Millipore, United States.

SWCNTs were synthesized by the arc discharge method utilizing graphite electrodes and Ni/Y (4/1 atomic \%) metal catalyst mixtures. Air oxidation of the SWCNT sample was performed in an oven at $350^{\circ} \mathrm{C}$ for two hours; a weight loss of $40 \sim 50 \%$ was observed during this process. As-grown, as well as air-oxidized nanotubes were dispersed ultrasonically in aqueous $1 \%$ SDBS (initial nanotube concentration $4 \mathrm{mg} / \mathrm{ml}$ ) and centrifuged at $13000 \mathrm{rpm}$ for $30 \mathrm{~min}$ (Hermle Z383, Germany) in order to increase their purity and decrease their metal content. The supernatant was decanted and the final concentration of nanotubes was estimated by UV-Vis spectroscopy using absorbance at $600 \mathrm{~nm}$ (Shimazdu UV2401PC, Japan). For the construction of the calibration line, dilutions from the unpurified dispersions were used with a well-known concentration. The relative purity of the nanotubes in suspension was determined from near infrared (NIR) spectra (Bruker Vertex70, Germany, spectrometer). The NIR purity index was calculated by comparing the baseline-corrected peak area corresponding to the interband $\mathrm{S}_{22}$ transition for the semiconducting nanotubes with the total area under the peak, as described in reference [33]. 
FTO substrates (SOLEMS ASAHI, Palaiseau, France, 70-100 $\Omega / \mathrm{sq}, 80 \mathrm{~nm}$ thickness) were degreased by sonicating them sequentially in isopropanol, acetone and ultrapure water for 1 min each prior to electrochemical measurements.

Polymerization of pyrrole on the FTO-coated glass substrate was performed by CV between -1.0 and $0.8 \mathrm{~V}$ vs. $\mathrm{Ag} / \mathrm{AgCl}(\mathrm{NaCl} 3 \mathrm{M}), 30$ cycles, with a sweep rate of 50 $\mathrm{mV} / \mathrm{s}$. Electropolymerization was performed in a $0.1 \mathrm{M}, \mathrm{pH}=6$ phosphate buffer solution containing $1 \%$ pyrrole $(0.14 \mathrm{M})$ in the case of Ppy-PO4 film, and 1\% pyrrole $+0.1 \%$ SDBS $\left(3.2 \cdot 10^{-3} \mathrm{M}\right)$ for the rest. The amount of nanotubes in the polymerization medium varies from 0 to $0.13 \mathrm{mg} / \mathrm{ml}$ for the different films. $\mathrm{An} \mathrm{Ag} / \mathrm{AgCl}(3 \mathrm{M} \mathrm{NaCl})$ electrode was used as a reference electrode, and a graphite bar was used as a counter electrode. For the electrochemical characterization of the film, $\mathrm{CV}$ was used at $5 \mathrm{mV} / \mathrm{s}$ and electrochemical impedance spectroscopy at -0.75 and $0.25 \mathrm{~V}$ vs. $\mathrm{Ag} / \mathrm{AgCl}$. The employed electrolyte was a $0.1 \mathrm{M}, \mathrm{pH}=6$ phosphate buffer solution. Potential limits for the cyclic voltammograms were -1.0 and $0.3 \mathrm{~V}$ to prevent the polymer overoxidation that can occur at higher potentials. Conditions for EIS were $\Delta \mathrm{E}=10 \mathrm{mV}$ and a frequency range from $10^{5}$ to $0.01 \mathrm{~Hz}$. Electrolytes were de-oxygenated employing high purity nitrogen to create an inert atmosphere. All the electrochemical measurements were performed using an AUTOLAB PGSTAT302N (Ecochemie B.V, The Netherlands) potentiostat. Zeta potential measurements were carried out using a Zetasizer Nano ZS (Malvern Instruments Ltd, United Kingdom).

AFM measurements were performed with a Multimode SPM from Veeco Instruments (Santa Barbara, US), equipped with Nanoscope V controller and JV-scanner $(10 \mu \mathrm{m}$ 
scan size in $\mathrm{XY}$, and $2.5 \mu \mathrm{m} \mathrm{Z}$-range. The system included the HarmoniX option which allows force-distance curves to be acquired in real time during tapping mode operation, and to mechanical properties to be extracted and mapped as additional data channels [34]. Soft silicon tapping mode cantilevers with off-axis tip design and reflective backside Al-coating (tip radius $10 \mathrm{~nm}$, spring constant $4 \mathrm{~N} / \mathrm{m}$, fundamental vertical resonance $70 \mathrm{kHz}$, torsional resonance $1200 \mathrm{kHz}$ ) were used that were optimized for large bandwidth acquisition of the force distance data (HMX, Veeco Probes, Camarillo, US). The probes were mounted in a cantilever holder with large dither piezo (model MFMA, Veeco Instruments, Santa Barbara, US). Ppy films were directly deposited on the FTO. Calibration of the modulus was performed by comparing the HarmoniX-data with a polymer sample of known modulus in the expected range; however, the stiffness values obtained are presented as semiquantitative and are used to compare the stiffness of the different films.

Scanning Electron micrographs were obtained using a Hitachi S-3400 N SEM EDX microscope. Optical confocal microcopy pictures and roughness profiles were taken using a Sensofar Pl $\mu 2300$ microscope.

\section{Results}

\subsection{Characterization of SWCNT dispersions}

The concentration and purity of the nanotubes were determined for both the as-grown and air-oxidized SWCNTs. Obtained NIR purity Index was 0.106 for the as-grown SWCNTs, and 0.258 in the case of air-oxidized SWCNTs. Although the NIR purity 
index does not give exact information about the absolute purity of the SWCNT sample, because of non-linearities in the absorbance [35] and the lack of a reference for $100 \%$

purity [36], it is a quantification of relative purity. Therefore, the purity of oxidized SWCNT dispersion is much higher than that of as-grown nanotubes. This is the same effect observed and described in [30], and it is explained by a preferential stabilization of the air-oxidized SWCNTs over the carbonaceous impurities present in the dispersion. This preferential stability is improved by the presence of anhydride and lactone groups on the surface of the SWCNTs. For the sake of comparability of the results between oxidized and as-grown nanotubes, two concentrations of the latter were used in the polymerization dispersion, $0.13 \mathrm{mg} / \mathrm{ml}$ and $0.018 \mathrm{mg} / \mathrm{ml}$, the lowest concentration being the same as the concentration of oxidized SWCNTs.

\subsection{Electrochemical polymerization}

Two regions can be distinguished in the electropolymerization voltammograms (Figure 1). The polymerization of pyrrole takes place at higher potentials $(>0.4 \mathrm{~V})$, while the doping-undoping of the polymer can be observed at lower potentials $(<0.4 \mathrm{~V})$. The onset potential of the polymerization is higher in the first cycle for all the synthesized films, indicating the presence of nucleation and growth processes during the initial stages of Ppy electrodeposition [10].

The presence of $0.1 \%$ SDBS in solution enhances the pyrrole polymerization process. This can be clearly seen when comparing the CVs in Figure 1A and Figure 1B; the current at $0.8 \mathrm{~V}$ is about 5 times greater in the latter case. The shape of the voltammetric profiles in Figure 1B is quite similar to those obtained by Fernández-Otero et al. [37]. A 
single, broad oxidation peak and a sharper reduction peak appear in the volttammogram, and peak separation increases as the experiment progresses.

The enhancement of the electropolymerization process is due to the surfactant effect of the DBS anion, which improves the wetting of the electrode surface by the solution [38]. In addition, DBS favors the transport of pyrrole towards the anode inside negatively-charged micelles [31]. Also, the doping effect of the added anion is supposed to increase the conductivity of the film, thus favoring further growth of the polymer layer. Furthermore, the current in the doping-undoping process region is much higher in the case of SDBS-Ppy electrodeposition, indicating a much thicker polymer film; this is in good concordance with an improvement in the electrical properties of the electrodeposited Ppy.

With regard to the co-deposition of the highest concentration carbon nanotubes $(0.13$

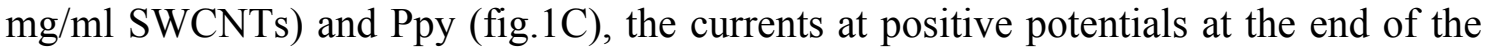
experiment $\left(30^{\text {th }}\right.$ cycle $)$ are higher in this case than in Figure 1B (ca. twice). Moreover, whereas, in the case of Ppy-SDBS, the current at $0.8 \mathrm{~V}$ decreases as the number of cycles advances, the situation is the reversed in the case of Ppy- $0.13 \mathrm{mg} / \mathrm{ml} \mathrm{SWCNTs}$, with the current at 0.8 increasing with the number of cycles.

Figures 1D and 1E show the formation of the films with low concentration of nanotubes, Ppy-0.018 $\mathrm{mg} / \mathrm{ml}$ agSWCNTs and Ppy-0.018 $\mathrm{mg} / \mathrm{ml}$ oxSWCNTs, respectively. The curves are very similar to each other. This is somewhat surprising, since oxidized SWCNTs and as-grown SWCNTs should exhibit very different surface chemical and electrochemical properties owing to the presence of oxygenated functional 
groups in the former [39]. The explanation for this effect is that the adsorption of the negatively charged DBS anion on agSWCNTs and oxSWCNTs provides a similar surface structure for both of them, making their electrochemical properties quite similar. The measured zeta potential was $-70.1 \pm 3.6 \mathrm{mV}$ for a $0.018 \mathrm{mg} / \mathrm{ml}$ agSWCNT, and $66.6 \pm 3.1 \mathrm{mV}$ for a $0.018 \mathrm{mg} / \mathrm{ml}$ oxSWCNT suspension, this indicates a quite similar surface structure, whose properties are determined by the adsorbed SDBS. The voltammograms in the doping-undoping region are very similar to those obtained for the Ppy-SDBS films. However, the current increases slightly with the number of cycles in the electropolymerization region, although the rise in the current is less noticeable than for the Ppy-0.13 mg/ml film.

A tentative explanation for this effect is that there is an increase in the electroactive area as nanotubes are deposited on the substrate. Negatively charged, SDBS-covered, SWCNTs are, by Brownian motion, and/or electrophoretically (due to the above mentioned negative surface charge), attached to the positively charged electrode surface. Once the nanotubes are stuck on the surface, Ppy electrodeposition takes place on their surface, thus increasing overall electroactive area. The number of nanotubes deposited is a function of the nanotube concentration and of the number of cycles; a higher number of cycles and a higher concentration will lead to a higher amount of deposited SWCNTs and, thus, a higher electropolymerization current. This statement will be examined in sections 3.3, 3.4 and 3.5.

\subsection{Electrochemical characterization}

\subsubsection{Cyclic Voltammetry}


Cyclic voltammograms for the different synthesized Ppy and composite films are shown in Figure 2. To obtain these voltammograms, a lower sweep rate $(5 \mathrm{mV} / \mathrm{s})$ was used in order to avoid the effects of mass transport limitations. The currents are much higher for the electrodes synthesized in the presence of SDBS than for Ppy synthesized with no additive, which is in good agreement with the voltammetric features obtained from the electropolymerization. Another interesting characteristic that can be observed is that voltammetric peaks for the doping-undoping process are significantly shifted towards negative potentials in the case of films synthesized in the presence of SDBS, which is due to the different mechanisms of this reaction; while in the Ppy without additives, phosphate anions are exchanged between the film and the electrolyte. In the case of Ppy-SDBS films, DBS anions are fixed in the polymer structure and $\mathrm{Na}^{+}$cations are exchanged between the electrolytic medium and the polymer [40], in this way favoring the oxidative doping of the polymer at lower potentials.

There is no dramatic difference between the cyclic voltammograms for the films synthesized in the presence of SDBS that are shown in Figure 2. The presence of nanotubes makes the curves behave more irreversibly; i.e., the potential difference between anodic and cathodic peaks is larger. This irreversibility is higher when agSWCNTs are added, and increases with the nanotube concentration.

The observed reversibility of the reaction follows the trend $0.1 \%$ SDBS $>0.018 \mathrm{mg} / \mathrm{ml}$ oxSWCNTs $>0.018 \mathrm{mg} / \mathrm{ml} \mathrm{agSWCNTs}>0.13 \mathrm{mg} / \mathrm{ml}$ agSWCNTs, but the differences are very slight, so a deeper study using EIS is required. The only significant difference in these films is the additional oxidation wave that appears at potentials between -0.05 
and $0.3 \mathrm{~V}$ for the Ppy- $0.13 \mathrm{mg} / \mathrm{ml}$ agSWCNTs film in the anodic sweep. This feature can be tentatively explained as Ppy polymerized in the presence of lower amounts of DBS anions near the electrode surface, this low concentration being due to diffusion problems within the pores. According to the previous literature, experiments carried out at SDBS concentrations lower than the critical micellar concentration show that SDBS at these low concentrations produces films with a much slower cation interchange rate [31].

\subsubsection{Electrochemical Impedance Spectroscopy}

Impedance measurements were carried out at two different potentials: $-0.75 \mathrm{~V}$, where the polymer film in the undoped state; and $0.25 \mathrm{~V}$, where the polymer is in a conducting doped state.

In order to acquire information from electrochemical impedance spectroscopy, the data must be fitted to an equivalent circuit. In our case, the model used to describe the behavior of the Ppy film is a slight modification of that shown in $[41,42]$. The model, shown in Figure 3, consists of a solution resistance $R_{\text {sol }}$, in series with a parallel combination of the capacitance $C P E_{c}$, and the charge-transfer resistance $R_{c t}$. In series with the last combination, the capacitance of the polymer film $C P E_{f}$, in parallel with a series combination of the resistance $R_{d}$, adscribed to the doping-undoping process of the polymer, and the Warburg impedance $Z_{w}$, which is related to the diffusion of ionic species within the film structure. When ionic diffusion within the film is fast enough, $Z_{w}$ is not taken into account and the equivalent circuit is simplified to one without the Warburg impedance element. 
$C P E_{c}$ is related either to the capacitance of the Ppy/FTO interface or of the Ppy/solution interface, modeled as a constant-phase element (CPE, an element used to describe the behavior of non-ideal capacitors [43]). $R_{c t}$ can be related either to the ionic charge transfer resistance at the polymer-solution interface or to the electron transfer in the FTO-Ppy interface [41] Film capacitance, $C P E_{f}$ is modeled also as a constant phase element

The parameters obtained by fitting the impedance spectra obtained at $0.25 \mathrm{~V}(\mathrm{Ag} / \mathrm{AgCl})$ to the model shown in Figure 3 are given in Table 1, and real capacitance values for the different films are shown in Table 2. The Nyquist plots obtained for the materials and the fitted curves are compared in Figure 4. The following expression was used to obtain those capacitance values from CPE parameters, which can be found in [44, 45]:

$C=\left(Y_{0}^{-1} R^{\alpha}\right)^{1 /(1-\alpha)}$

where $C$ is the calculated capacitance and $R$ is the value of the resistance in parallel with the CPE.

Variations in the values for $R_{\text {sol }}$ are assigned to cell configuration conditions. In every different experiment there are slight changes in the position of either the reference electrode, or the working electrode, or both. The distance between electrodes is in the range of a few milimeters in order to minimize this $R_{\text {sol }}$. So, these slight changes strongly affect the value of solution resistance. This parameter could also be affected by film properties, such as electronic conductivity, which is, in turn affected by other 
properties, such as, quantity SWCNTs, thickness or porosity. However, there is no observed correlation between those properties (see 3.4 and 3.5) and the obtained $R_{\text {sol }}$.

The values of $C_{c}$ obtained for the different films synthesized in the presence of SDBS are too similar to one another to be ascribed to the polymer-solution interface, given that morphologies of the films are totally different, (see 3.4 and 3.5). Those values are more likely related to the capacitance of the polymer-FTO interface, since the current values in the first cycles of electropolymerization are similar (figures $1 \mathrm{~B}$ to $1 \mathrm{E}$ ). This indicates that the first polymer layers in contact with the electrode are similar. Also, the resistance $R_{c t}$ decreases in the presence of SDBS, which is ascribed to a better adherence of the polymer film to the substrate. These resistance values increase when the SWCNT concentration increases, pointing to an influence of the nanotube-polymer or the nanotube-FTO interface on this system.

With regard to the low-frequency loop, it is not possible to obtain a good fit between the proposed models and the experimental results in the case of Ppy- $\mathrm{PO}_{4}$ films $\left(R_{d}\right.$ turns out to be infinity). This is due to the completely different nature of the process taking place in the film. In the SDBS synthesized films, the capacitance of the films $C_{f}$ is dramatically increased by the presence of carbon nanotubes. Observing these results, the contribution of the capacitance of the electrode-solution interface seems to be indistinguishable from the intrinsic film capacitance. The influence of SWCNT concentration is more significant than the influence of nanotube type, as can be seen in table 2. These huge differences may be explained using two contributions. While a smooth, thin film is obtained in the absence of carbon nanotubes, thicker and rougher films are obtained in the case of nanotube containing deposits (sections 3.4 and 3.5). 
These capacitance values are the explanation for the differences in impedance modulus observed at low frequencies.

Differences in $R_{d}$ are also found. This resistance is significantly higher in the Ppy-0.13 $\mathrm{mg} / \mathrm{ml}$ agSWCNTs electrodeposited film, which is in agreement with our statement in 3.3.1. that the polymerization of pyrrole in lower concentrations of SDBS would give rise to films with slower cation exchange kinetics [31]. Another feature concerning this parameter is that it is particularly low in the case of Ppy- $0.018 \mathrm{mg} / \mathrm{ml} \mathrm{agSWCNTs}$. The origin of this nanotube type effect is likely to be due to a more positive half-wave potential $\left(E_{1 / 2}\right)$ for the doping-undoping of Ppy-0.018 mg/ml SWCNT film [43].

As for the diffusion admittances $Y_{0}\left(Z_{w}\right)$, the biggest difference is observed between 0.13 $\mathrm{mg} / \mathrm{ml}$ agSWCNT film, which shows no diffusion impedance, and the rest, which show values quite similar to one another. The exceptionally higher values for the Ppy-0.13 $\mathrm{mg} / \mathrm{ml}$ agSWCNT films can be explained by the high $R_{d}$ obtained for this film, making the ion diffusion limitations less important compared to the electronic transfer limitations.

For use of Ppy films in neural interfaces, corrected electrode impedance modulus, calculated by subtracting the solution resistance from the real part of the impedance, of the system, was compared in a frequency range between $100 \mathrm{~Hz}$ and $1000 \mathrm{~Hz}$ (figure 5). At the frequency of $1 \mathrm{kHz}$, the standard used in the literature [46], the addition of SDBS significantly lowers the electrode impedance modulus of the Ppy films. In addition to the biocompatibility of the SDBS, this change makes this material a promising substrate for cell growth. The addition of a small quantity of carbon nanotubes does not 
substantially affect the impedance modulus values. Since $1000 \mathrm{~Hz}$ is located in the high frequency loop, this increase in the impedance modulus is ascribed to a slow electronic transfer between the nanotubes and either the Ppy film or the FTO substrate, as previously mentioned. Despite their non-influence on impedance modulus at $1 \mathrm{kHz}$, the adding of SWCNTs to the films improves morphological and mechanical properties (3.4 to 3.6). Those properties make them good candidates for cell growth substrates. More work is in progress in this direction.

\subsection{SEM micrographs}

SEM images show significant differences between the morphology of the films synthesized in different conditions (Figure 6). Ppy films (Figure 6A) with no additive form very thin films that follow the topography of the FTO substrate; Ppy-SDBS shows a very compact and smooth surface structure; and Ppy-agSWCNT films show an open, porous structure. This is consistent with the cyclic voltammograms obtained in 3.3.1. As for the PPy-SWCNT layers (fig 6 C-E), a rougher structure can be seen. No significant differences are observed between Ppy-0.018 mg/ml agSWCNTs (fig 6D) and Ppy-0.018 $\mathrm{mg} / \mathrm{ml}$ oxSWCNTs. This is coherent with the electrochemical properties observed in sections 3.2 and 3.3. In the case of Ppy- $0.13 \mathrm{mg} / \mathrm{ml}$ agSWCNTs (fig 6C), a more closely packed structure with smaller pore size is observed. These observations are coherent with the electrochemical results.

\subsection{Confocal microscopy results.}


The film thickness and surface roughness of the samples were examined using confocal microscopy, a technique that contains three-dimensional information that SEM cannot provide. Table 3 shows the values of the average film thickness $t$ and arithmetical mean roughness $R_{a}$, which is the mean deviation of the absolute value of the "real" film thickness with respect to the average film thickness, for all synthesized films. The film synthesized in the absence of SDBS is much thinner than the rest (more than one order of magnitude). This is in agreement with the cyclic voltammetry results, which show significantly lower currents for the electrodeposition and the doping-undoping process in this case (see 3.3). With regard to film roughness, in both cases this is similar and very low. A thicker film is also observed in the presence of carbon nanotubes: the thickness of the films increases as the concentration of SWCNTs increases, but the film roughness decreases as the concentration increases. This is, in principle, a surprising result, but it is due to the fact that the lateral resolution of the technique $(\sim 500 \mathrm{~nm})$ is not high enough to distinguish small pores from a smooth surface, thus giving $R_{a}$ values that are lower than the real ones. Also, the thickness of the Ppy- $0.018 \mathrm{mg} / \mathrm{ml} \mathrm{agSWCNT}$ film is slightly higher than that of Ppy- $0.018 \mathrm{mg} / \mathrm{ml}$ oxSWCNT film, this is in good agreement with the measured zeta potential, which is also higher for the Ppy-0.018 $\mathrm{mg} / \mathrm{ml}$ agSWCNT film, thus suggesting an influence of the electrophoretic motion of the SWCNTs towards the electrode in the electropolymerization process.

\subsection{AFM measurements}

Topographic information can be completed using AFM measurements. This technique has the advantage of a very good lateral resolution but, on the other hand, it has the disadvantages of a narrower scan XY window and a limited displacement along the Z- 
axis. However, it does give crucial information about the mechanical properties of the films, information that Confocal Microscopy is unable to provide.

Figures 7A and 7B show the AFM images corresponding to the topography and the stiffness map of the Ppy-0.1\% SDBS films, while Figures 7C and 7D show the topography and the stiffness map of the Ppy- $0.13 \mathrm{mg} / \mathrm{mL}$ agSWCNT films. The topography of the Ppy-SDBS films shows a globular structure, with the stiffness

mapping showing a homogeneous distribution. At the same time, there is a nanostructure consisting of two closely mixed components of different stiffnesses corresponding to the Ppy and SDBS. The topography image corresponding to the Ppy$0.13 \mathrm{mg} / \mathrm{mL}$ agSWCNT film (Figure 7C) shows a less homogeneous distribution than the Ppy- $0.1 \%$ SDBS film. Small circular areas can be seen that present less stiffness and which could consist of Ppy without SWCNTs, or areas where the composite has not been produced. The stiffness of this small area is approximately the same as the Ppy$0.1 \%$ SDBS films and less than that of the rest of the Ppy- $0.13 \mathrm{mg} / \mathrm{mL}$ agSWCNTs films, showing that the presence of SWCNTs in the film improves the mechanical properties of the Ppy.

\section{Conclusions}

The effect of SDBS and the SDBS-dispersed nanotubes on the electropolymerization of pyrrole on transparent FTO substrates has been studied using electrochemical and exsitu techniques. CV has been used for the synthesis and characterization of the films, and EIS spectra have been recorded and fitted to a model for conductive polymers. SDBS acts, simultaneously, as a stabilizer of the nanotube dispersion, as a nanotube 
purifier and as a dopant anion, allowing the use of any type of nanotube of controlled purity, otherwise not possible. The addition of SDBS dramatically increases the values of an electropolymerization current. The type of nanotubes has little effect on this current, but its value rises when SWCNT concentration arises. CV characterization properties of the films are mainly controlled by the presence of SDBS, which increases the capacitance of the film and lowers the impedance modulus between 300 and 1000 $\mathrm{Hz}$, with the effect of the nanotubes being less important. The observed effect on Ppy$0.13 \mathrm{mg} / \mathrm{ml}$ agSWCNTs at high potential is attributed to transport limitation during the electropolymerization process owing to the porosity of the SWCNT-Ppy structure. The presence of SWCNTs strongly affects the morphology and structure of the film, making films rougher and thicker. Surface roughness and film thickness affect the parameters obtained using EIS, giving rise to a dramatic increase in the film capacitance when nanotube concentration increases. This capacitance also increases when oxSWCNTs are employed with respect to the agSWCNTs of the same concentration. The stiffness of the Ppy-0.13 mg/ml agSWCNT film, obtained by AFM, resulted higher than that of the Ppy- $0.1 \%$ SDBS film, showing that the presence of nanotubes improves the mechanical properties of the films.

\section{Acknowledgements}

The authors wish to thank Spanish Ministry of Science and Innovation (MICINN) and the European Regional Development Fund (ERDF) for financial support under project No REF EUI2008-00153, the Government of Aragon (DGA) for funding under projects DGA-T66 CNN and DGA P108/09, and Mercedes Vico-Gallardo for her dedicated and 
helpful work. J. Hernández-Ferrer and A. Ansón-Casaos acknowledge the Spanish Superior Council for Scientific Research (CSIC) for their JAE-Doc contracts.

[1] J.r. Heinze, B.A. Frontana-Uribe, S. Ludwigs, Chem. Rev., 110 (2010) 4724-4771.

[2] G.A. Snook, P. Kao, A.S. Best, J. Power Sources, 196 (2011) 1-12.

[3] A.D. Bendrea, L. Cianga, I. Cianga, Journal of Biomaterials Applications, 26 (2011) 3-84.

[4] Q. Li, J. Wu, Z. Tang, Y. Xiao, M. Huang, J. Lin, Electrochim. Acta, 55 (2010) 2777-2781.

[5] T. Makris, V. Dracopoulos, T. Stergiopoulos, P. Lianos, Electrochim. Acta, 56 (2011) 2004-2008.

[6] H. Nagai, H. Segawa, Chemical Communications, 10 (2004) 974-975.

[7] V. Brânzoi, L. Pilan, F. Golgovici, F. Brânzoi, Molecular Crystals and Liquid Crystals, 446 (2006) 305-318.

[8] I.L. Lehr, S.B. Saidman, Corrosion Science, 49 (2007) 2210-2225.

[9] M. Holzinger, Nonlinear Optics Quantum Optics, 39 (2009) 23-39.

[10] S. Sadki, P. Schottland, N. Brodie, G. Sabouraud, Chem. Soc. Rev., 29 (2000) 283293.

[11] A. Mohammadi, Y. Yamini, N. Alizadeh, Journal of Chromatography A, 1063 (2005) 1-8.

[12] J. Tamm, T. Raudsepp, M. Marandi, T. Tamm, Synth. Met., 157 (2007) 66-73.

[13] T.F. Otero, E. De Larreta, Synth. Met., 26 (1988) 79-88.

[14] Z. Spitalsky, D. Tasis, K. Papagelis, C. Galiotis, Progress in Polymer Science, 35 (2010) 357-401.

[15] A.M. Diez-Pascual, G. Martinez, J.M. Gonzalez-Dominguez, A. Anson, M.T.

Martinez, M.A. Gomez, J. Mater. Chem., 20 (2010) 8285-8296.

[16] J.M. González-Domínguez, A. Ansón-Casaos, A.M. Díez-Pascual, B. Ashrafi, M.

Naffakh, D. Backman, H. Stadler, A. Johnston, M. Gómez, M.T. Martínez, ACS App.

Mater. Interfac., 3 (2011) 1441-1450.

[17] Y.-W. Lin, T.-M. Wu, Polymer International, 60 (2011) 382-388.

[18] H. Chen, L. Guo, A.R. Ferhan, D.H. Kim, J. Phys. Chem. C, 115 (2011) 54925499.

[19] H. Durgam, S. Sapp, C. Deister, Z. Khaing, E. Chang, S. Luebben, C.E. Schmidt, Journal of Biomaterials Science, Polymer Edition, 21 (2010) 1265-1282.

[20] A. Kotwal, C.E. Schmidt, Biomaterials, 22 (2001) 1055-1064.

[21] M.R. Abidian, D.C. Martin, Biomaterials, 29 (2008) 1273-1283.

[22] R.A. Green, N.H. Lovell, G.G. Wallace, L.A. Poole-Warren, Biomaterials, 29 (2008) 3393-3399.

[23] Y. Lu, T. Li, X. Zhao, M. Li, Y. Cao, H. Yang, Y.Y. Duan, Biomaterials, 31 (2010) 5169-5181.

[24] J. Wang, Y. Xu, F. Yan, J. Zhu, J. Wang, F. Xiao, J. Solid State Electrochem., 14 (2010) 1565-1575.

[25] A. Ansón-Casaos, J.M. González-Domínguez, M.T. Martínez, Carbon, 48 (2010) 2917-2924. 
[26] H. Cathcart, J.N. Coleman, Chem. Phys. Lett., 474 (2009) 122-126.

[27] J.L. Blackburn, C. Engtrakul, T.J. McDonald, A.C. Dillon, M.J. Heben, J. Phys.

Chem. B, 110 (2006) 25551-25558.

[28] V.C. Moore, M.S. Strano, E.H. Haroz, R.H. Hauge, R.E. Smalley, J. Schmidt, Y.

Talmon, Nano Letters, 3 (2003) 1379-1382.

[29] R. Shvartzman-Cohen, E. Nativ-Roth, E. Baskaran, Y. Levi-Kalisman, I. Szleifer,

R. Yerushalmi-Rozen, J. Am. Chem. Soc., 126 (2004) 14850-14857.

[30] A. Ansón-Casaos, M.n. González, J.M. González-Domínguez, M.T. Martínez, Langmuir, 27 (2011) 7192-7198.

[31] K. Naoi, Y. Oura, M. Maeda, S. Nakamura, J. Electrochem. Soc., 142 (1995) 417422.

[32] A.R. Boccaccini, J. Cho, J.A. Roether, B.J.C. Thomas, E. Jane Minay, M.S.P. Shaffer, Carbon, 44 (2006) 3149-3160.

[33] M.E. Itkis, D.E. Perea, S. Niyogi, S.M. Rickard, M.A. Hamon, H. Hu, B. Zhao,

R.C. Haddon, Nano Letters, 3 (2003) 309-314.

[34] S. Ozgur, E. Natalia, Nanotechnology, 19 (2008) 445717.

[35] B.J. Landi, H.J. Ruf, C.M. Evans, C.D. Cress, R.P. Raffaelle, J. Phys. Chem. B,

109 (2005) 9952-9965.

[36] M.E. Itkis, D.E. Perea, R. Jung, S. Niyogi, R.C. Haddon, J. Am. Chem. Soc., 127 (2005) 3439-3448.

[37] F.J.G. Frutos, T.F. Otero, A.J.F. Romero, Electrochim. Acta, 52 (2007) 3621-3629.

[38] E.L. Kupila, J. Kankare, Synth. Met., 55 (1993) 1402-1405.

[39] H. Hu, A. Yu, E. Kim, B. Zhao, M.E. Itkis, E. Bekyarova, R.C. Haddon, J. Phys.

Chem. B, 109 (2005) 11520-11524.

[40] M.-A. De Paoli, R.C.D. Peres, S. Panero, B. Scrosati, Electrochim. Acta, 37 (1992) 1173-1182.

[41] A. Hallik, A. Alumaa, J. Tamm, V. Sammelselg, M. Väärtnõu, A. Jänes, E. Lust, Synth. Met., 156 (2006) 488-494.

[42] M.F. Suarez-Herrera, J.M. Feliu, Phys. Chem. Chem. Phys., 10 (2008) 7022-7030.

[43] A. Lasia, Electrochemical Impedance Spectroscopy and its Applications., in: B.E.

Conway, J.O.M. Bockris, R.E. White (Eds.) Modern Aspects of Electrochemistry, vol.

32, Kluwer Academics/Plenum Publishers, New York, 1999, pp. 143-248.

[44] C.H. Hsu, F. Mansfeld, CORROSION, 57 (2001) 747-748.

[45] G.J. Brug, A.L.G. van den Eeden, M. Sluyters-Rehbach, J.H. Sluyters, Journal of Electroanalytical Chemistry and Interfacial Electrochemistry, 176 (1984) 275-295.

[46] X. Cui, D.C. Martin, Sensors and Actuators B: Chemical, 89 (2003) 92-102. 
Table 1. Values for the parameters obtained when the EIS data for the obtained films are fitted to the equivalent circuit shown in figure 3. The meaning of the parameters is shown in the text (section 3.3.2)

\begin{tabular}{|c|c|c|c|c|c|c|c|c|}
\hline & $\mathrm{R}_{\mathrm{sol}} / \mathrm{W}$ & $\mathrm{R}_{\mathrm{ct}} / \mathrm{W}$ & $\mathrm{Y}_{0}\left(\mathrm{CPE}_{\mathrm{c}}\right) / \mathrm{S}$ & $\alpha\left(\mathrm{CPE}_{\mathrm{c}}\right)$ & $\mathrm{R}_{\mathrm{d}} / \Omega$ & $\mathrm{Y}_{0}\left(\mathrm{CPE}_{\mathrm{f}}\right) / \mathrm{S}$ & $\mathrm{a}\left(\mathrm{CPE}_{\mathrm{f}}\right)$ & $\mathrm{Y}_{0}\left(\mathrm{Z}_{\mathrm{w}}\right) / \mathrm{S}$ \\
\hline $\mathrm{Ppy}-\mathrm{PO}_{4}$ & 120.5 & 277.8 & $1.11 \mathrm{E}-04$ & 0.52699 & infinity & $2.01 \mathrm{E}-4$ & 0.76573 & infinity \\
\hline Ppy- $0.1 \%$ SDBS & 110.01 & 28.158 & $3.69 \mathrm{E}-04$ & 0.58 & 7032.6 & 7.24E-05 & 0.89 & $6.80 \mathrm{E}-04$ \\
\hline Ppy-0.13 mg/ml agSWCNTs & 140.48 & 247.64 & $5.24 \mathrm{E}-05$ & 0.78 & 14481 & $1.20 \mathrm{E}-03$ & 0.71 & infinity \\
\hline Ppy- $0.018 \mathrm{mg} / \mathrm{ml} \mathrm{agSWCNTs}$ & 157.04 & 89.269 & $5.55 \mathrm{E}-05$ & 0.82 & 4005.5 & 3.43E-04 & 0.85 & $8.16 \mathrm{E}-04$ \\
\hline Ppy- $0.018 \mathrm{mg} / \mathrm{ml}$ oxSWCNTs & 166.77 & 67.924 & $1.93 \mathrm{E}-04$ & 0.63 & 7876.5 & 4.69E-04 & 0.85 & $6.13 \mathrm{E}-04$ \\
\hline
\end{tabular}


Table 2. Capacitance of the obtained films. Values calculated using equation 2 . The meaning of $\mathrm{C}_{\mathrm{c}}$ and $\mathrm{C}_{\mathrm{f}}$ is shown in the text (section 3.3.2)

\begin{tabular}{|c|c|c|}
\hline $0.25 \mathrm{~V}$ & $\mathrm{C}_{\mathrm{c}} / \mathrm{F}$ & $\mathrm{C}_{\mathrm{f}} / \mathrm{F}$ \\
\hline Ppy-PO $\mathrm{PO}_{4}$ & $4.87 \cdot 10^{-6}$ & $\begin{array}{ll}--- \\
---\end{array}$ \\
\hline Ppy-0.1\% SDBS & $1.35 \cdot 10^{-5}$ & $6.66 \cdot 10-5$ \\
\hline Ppy-0.13 mg/ml agSWCNTs & $1.54 \cdot 10^{-5}$ & $3.85 \cdot 10-3$ \\
\hline Ppy-0.018 mg/ml agSWCNTs & $1.73 \cdot 10^{-5}$ & $3.63 \cdot 10-4$ \\
\hline Ppy-0.018 mg/ml oxSWCNTs & $1.51 \cdot 10^{-5}$ & $5.91 \cdot 10-4$ \\
\hline
\end{tabular}

Table 3. Average film thickness $t$ and arithmetical mean roughness $R_{a}$ obtained by Confocal Microscopy for the different electrosynthesized films.

\begin{tabular}{lcc}
\hline & $t / \mathrm{mm}$ & $R_{a} / \mathrm{mm}$ \\
\hline Ppy-PO $_{4}$ & 0.087 & 0.12 \\
& & \\
Ppy-0.1\% SDBS & 1.15 & 0.14 \\
Ppy-0.13 mg/ml agSWCNTs & 4.54 & 0.73 \\
& & \\
Ppy-0.018 mg/ml agSWCNTs & 3.37 & 1.36 \\
& & \\
Ppy-0.018 mg/ml oxSWCNTs & 2.77 & 1.35 \\
\hline
\end{tabular}


Figure 1
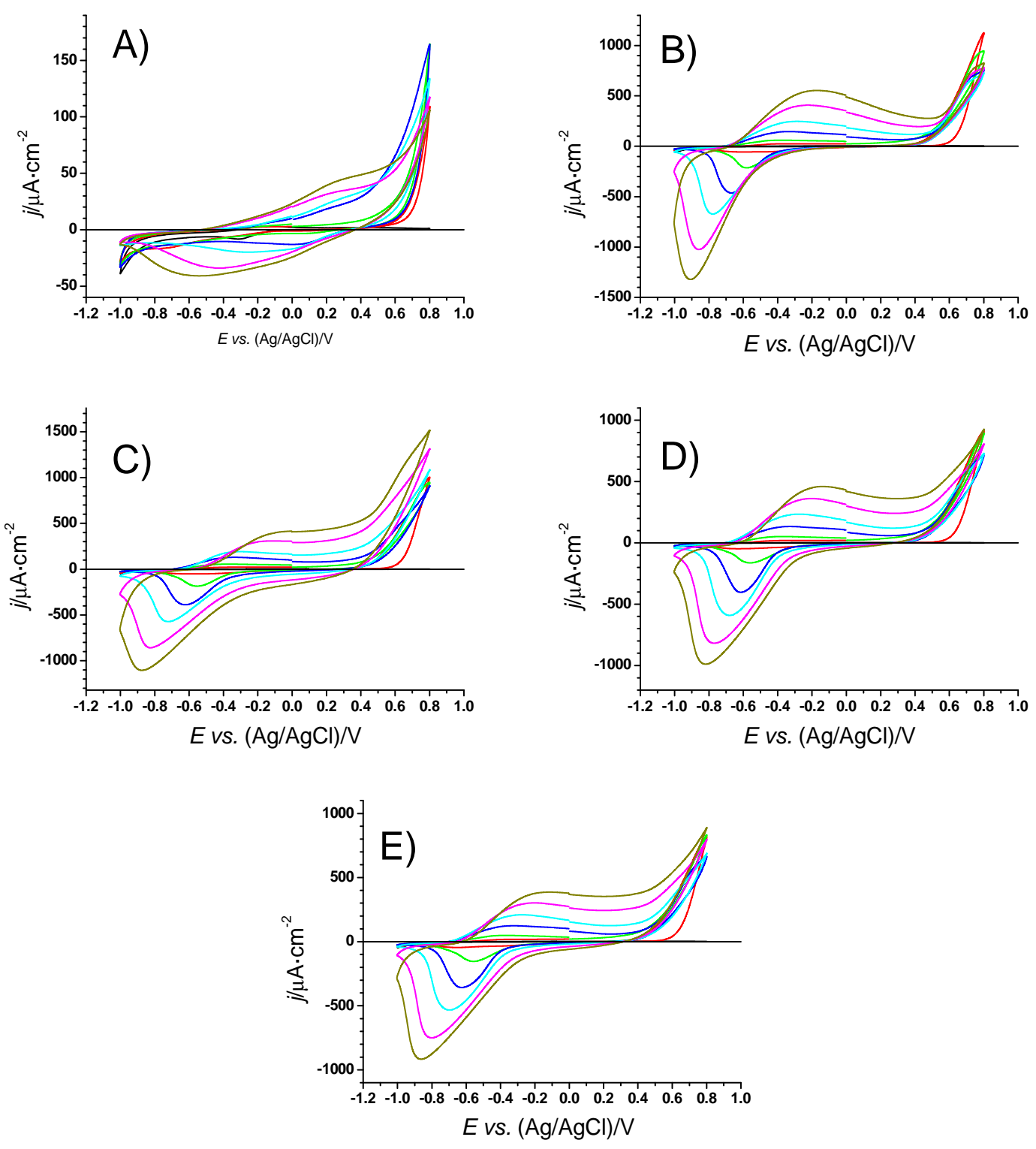

Figure 1. Cyclic voltammetries for the electropolymerization process on FTO for (a) Ppy-PO 4 , (b) Ppy-0.1\% SDBS, (c) Ppy-0.1\% SDBS-0.013\% agSWCNTs, (d) Ppy-0.018 mg/ml agSWCNTs, (e) Ppy $-0.018 \mathrm{mg} / \mathrm{ml}$ oxSWCNTs. FTO substrate (black), $1^{\text {st }}$ cycle (red), $2^{\text {nd }}$ cycle (green), $5^{\text {th }}$ cycle (blue), $10^{\text {th }}$ cycle (cyan), $20^{\text {th }}$ cycle (magenta), $30^{\text {th }}$ cycle (dark yellow). 
Figure 2

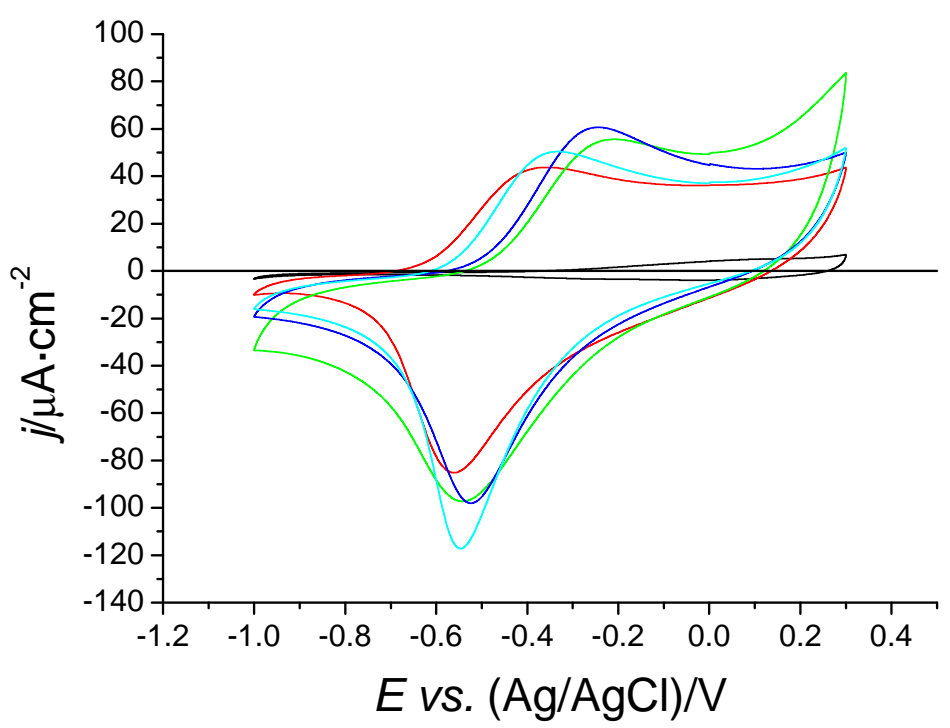

Figure 2. Cyclic voltammetries in $0.1 \mathrm{M} \mathrm{pH}=6$ phosphate buffer on FTO for Ppy-PO4 (black), Ppy-0.1\% SDBS (red), Ppy-0.13 mg/ml agSWCNTs (green), Ppy-0.018 mg/ml agSWCNTs (blue), Ppy-0.018 mg/ml oxSWCNTs (blue). a) sweep rate $=50 \mathrm{mV} \cdot \mathrm{s}^{-1}, \mathrm{~b}$ ) sweep rate $=5 \mathrm{mV} \cdot \mathrm{s}^{-1}$. 
Figure 3

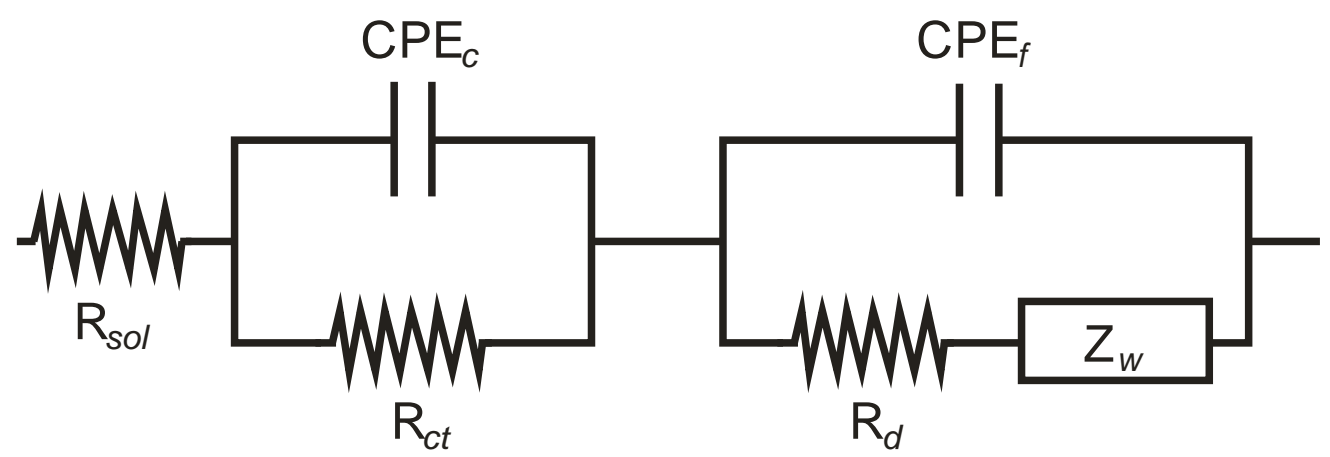

Figure 3. Scheme of the equivalent circuit used to model the electrochemical behavior of the electrodeposited films. The parameters exposed in the model are explained in the text (section 3.3.2). 
Figure 4

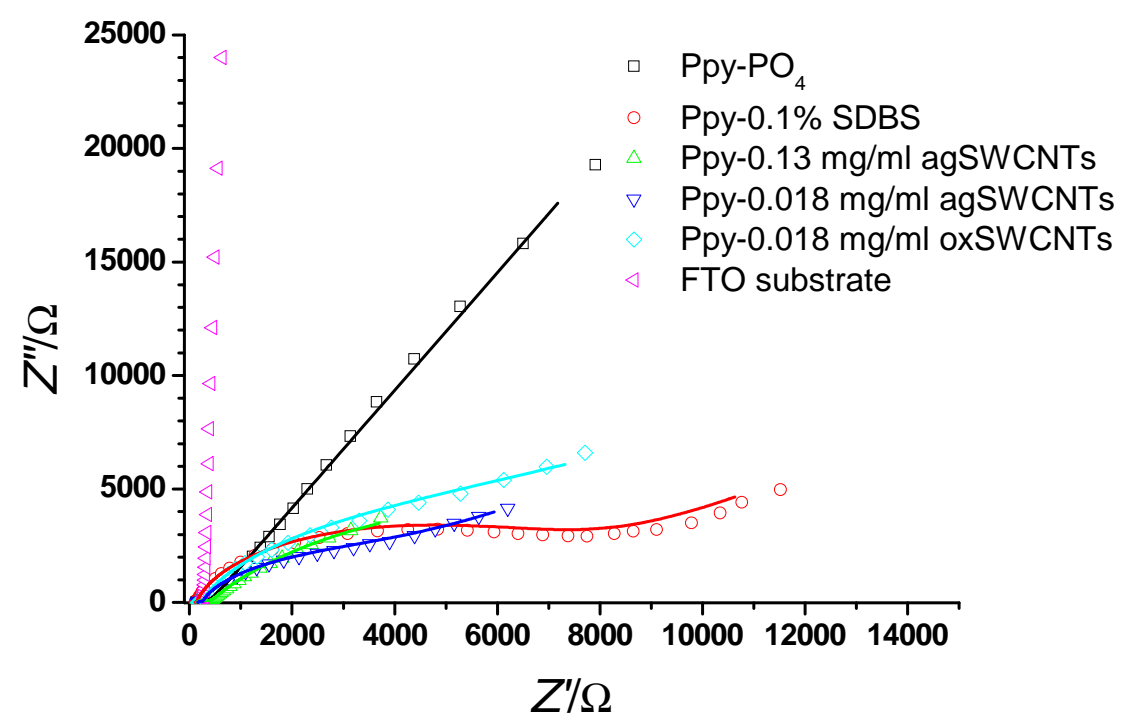

Figure 4. Nyquist plots of the impedances at $0.25 \mathrm{~V}$ (scatter graphs) for the different electrodes, and the fittings to the model proposed in 3.3.2. (solid lines). Legends within the graph. 
Figure 5

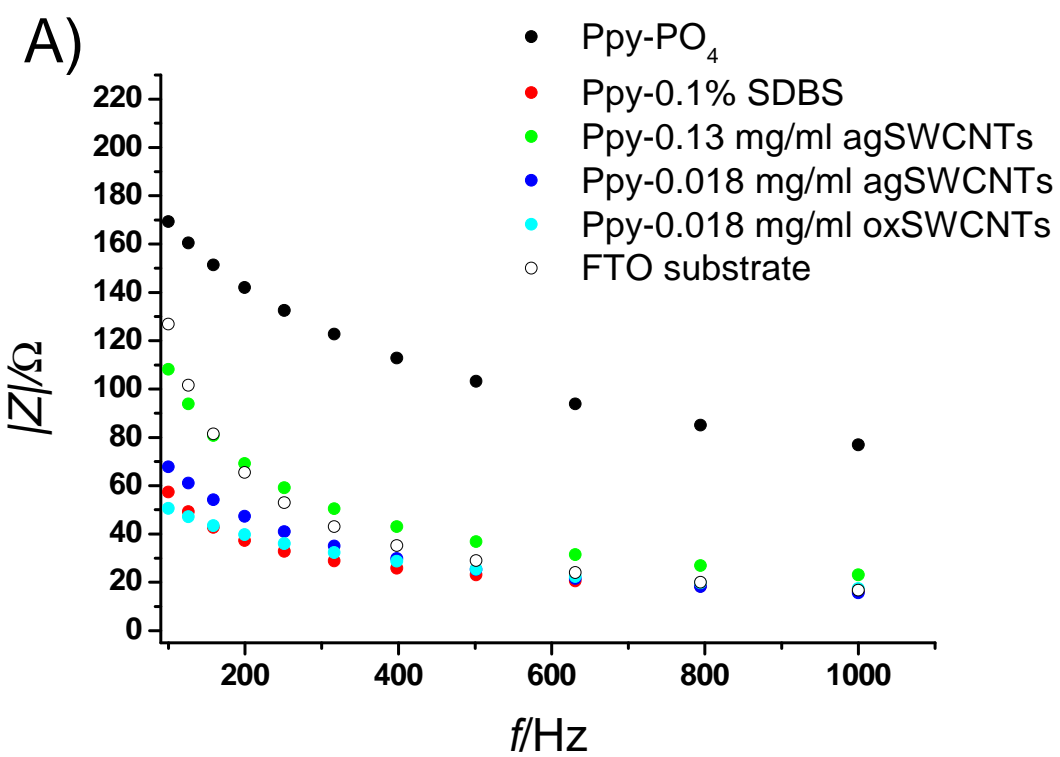

B)

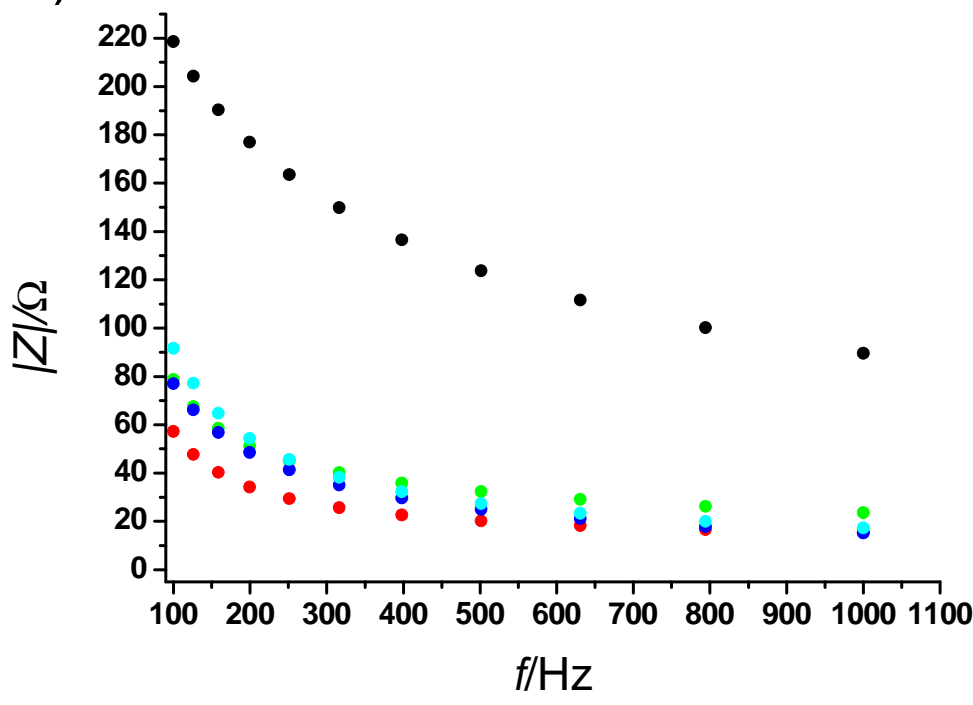

Figure 5. Comparison of the impedances between 100 and $1000 \mathrm{~Hz}$ for the electrodeposited films at A) $0.25 \mathrm{~V}$ and B) $-0.75 \mathrm{~V}$, legend is displayed within the graph. 
Figure 6
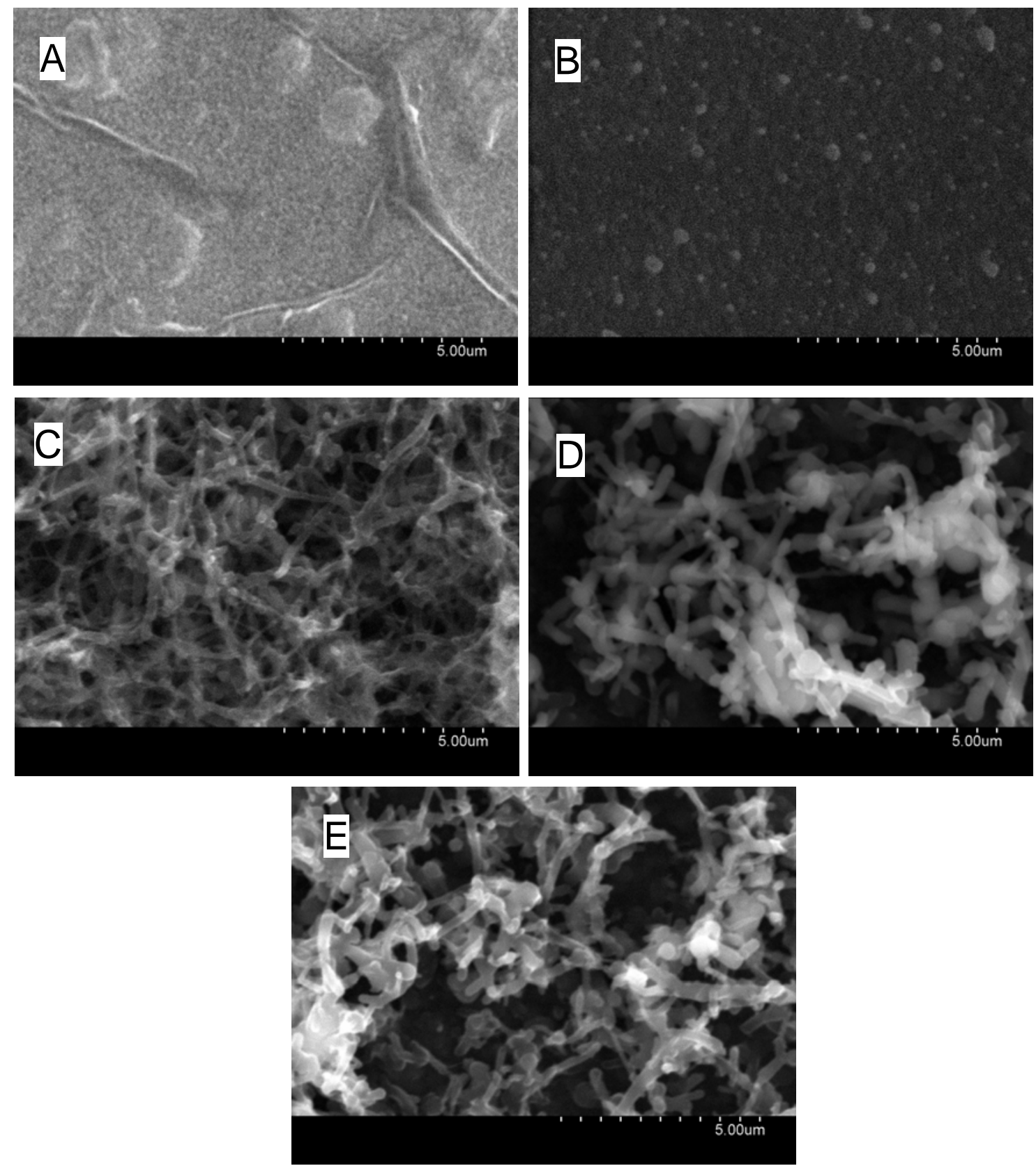

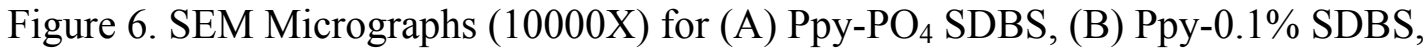

(C) Ppy $-0.13 \mathrm{mg} / \mathrm{ml}$ agSWCNTs, (D) Ppy-0.1\% SDBS-0.018 mg/ml agSWCNTs, (E)

Ppy-0.018 mg/ml oxSWCNTs. 
Figure 7

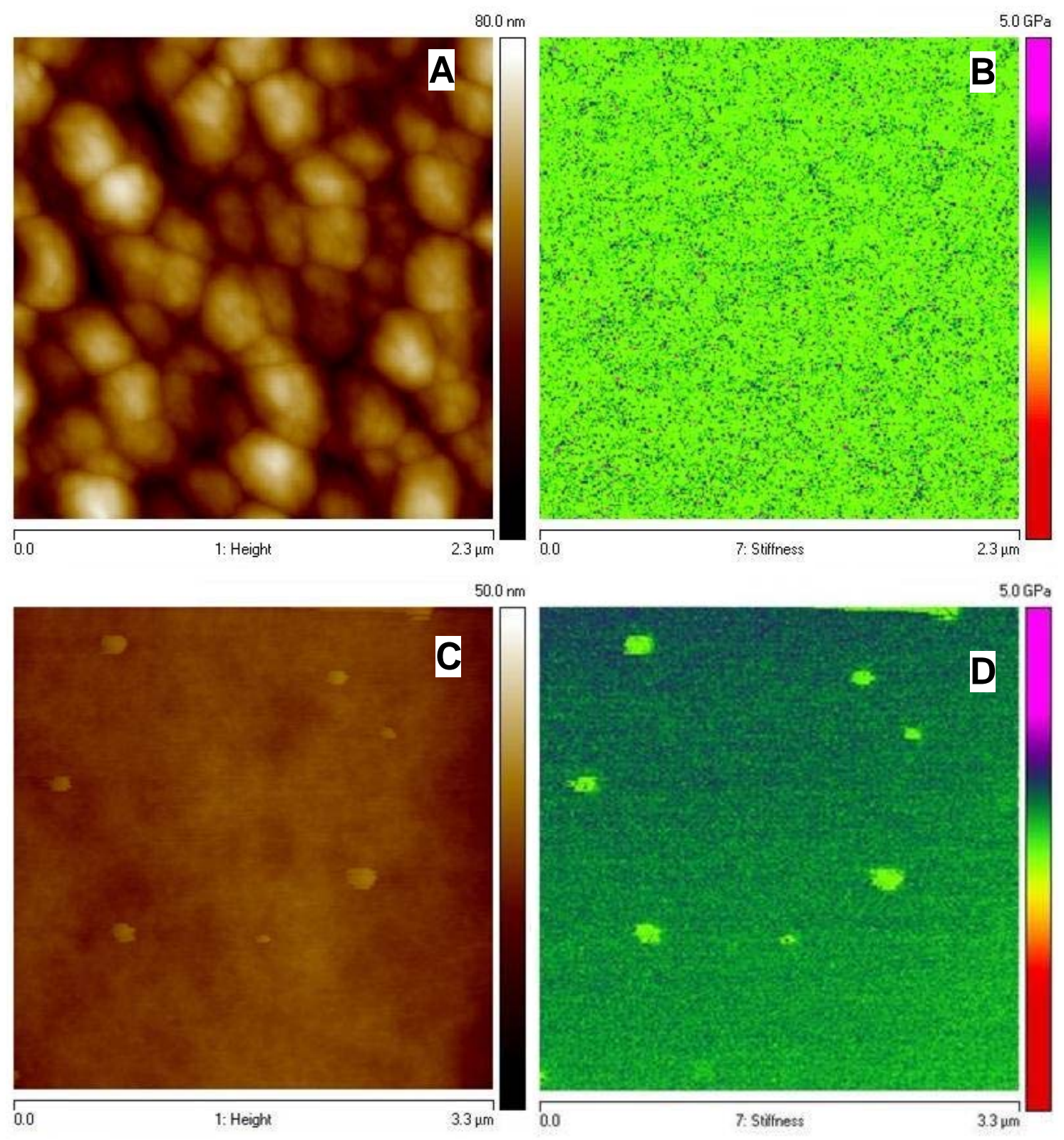

Figure 7. A and B, AFM images of the topography and the stiffness map of the Ppy$0.1 \%$ SDBS film. C and D topography and the stiffness map of the Ppy- $0.13 \mathrm{mg} / \mathrm{mL}$ agSWCNT film. 